\title{
Formulación en opciones reales para la asignación óptima de energía y reserva para una compañía de generación
}

\author{
Energy and reserve genco's self-scheduling: real option approach formulation
}

\author{
G.D. Mendoza-Mendoza ${ }^{1} \quad$ G. Gutiérrez-Alcaraz ${ }^{1}$
}

Recibido 6 de septiembre de 2010, aceptado 4 de junio de 2015

Received: September 6, 2010 Accepted: June 4, 2015

\begin{abstract}
RESUMEN
La nueva industria eléctrica está evolucionando en las actividades del mercado. Los primeros modelos de mercados solamente consideraban un producto negociable: energía. Todos los servicios auxiliares (SA), se agregaban o incluían en el precio de la energía. En la actualidad los SA están claramente definidos y en algunos mercados de electricidad, existen mercados simultáneos para algunos de los SA.

En un mercado de electricidad desagregado, las compañías de generación (GenCos) podrán participar en la prestación de diferentes servicios. En este trabajo, se consideran dos mercados simultáneos: energía y reserva rodante. La asignación óptima de servicios ofertados por una GenCo es formulado en un marco de opciones reales (OR). En el contexto de electricidad, el enfoque de OR puede usarse como un método para identificar y cuantificar las decisiones ante contingencias embebidas en la posesión de activos de generación, lo cual permite mejorar considerablemente la eficacia de las decisiones operativas. Las ofertas para reserva se presentan sobre una base diaria. Las ofertas consisten en un precio por capacidad y uno por energía.
\end{abstract}

Palabras clave: Asignación de unidades, mercados de electricidad, opciones reales, reserva de potencia activa, servicios auxiliares.

\begin{abstract}
The new electricity industry is evolving in market activities. The first electricity market models considering only one traded commodity: energy. All the supportive services, ancillary services (SA), were imputed in the energy price. Currently, the supportive services are clearly defined. In some electricity markets concurrent spot markets exist for some of the supportive services.

In an unbundled electricity market, Generation Companies (GenCos) may participate in the provision of different services. Two concurrent spot markets are considered in this paper: energy and spinning reserve. Optimal self-scheduling for a GenCo is formulated in a real options framework. In the context of electricity producing firms, real options approach (ROA) can be used as a method of identifying and quantifying the contingent decisions embedded in owning generation assets. It enhances the potential to improve greatly the effectiveness of operating decisions and to unlock hidden assets. The bids for reserve are submitted on a day-to-day basis. The bids consist of a capacity and energy price.
\end{abstract}

Keywords: Optimum unit commitment schedules, electricity market, real option analysis, reserve dispatch, ancillary service market.

1 Programa de Graduados e Investigación en Ingeniería Eléctrica. Instituto Tecnológico de Morelia. Av. Tecnológico 1500, Col. Santiaguito, C.P. 58120. Morelia Michoacán, México. E-mail: gidmem9@yahoo.com.mx; ggutier@itmorelia.edu.mx 


\section{INTRODUCCIÓN}

En general todos los modelos de mercados de electricidad buscan el diseño de un mercado eficaz, seguro y transparente, que optimice la programación y el despacho de potencia, maximizando el beneficio social y satisfaciendo todas las restricciones operativas del sistema eléctrico de potencia. Las actividades de procuración de energía y reserva, cálculo de precios, remuneración de productores y proveedores de servicios, así como la recuperación de costos, se fundamenta en una secuencia de procesos que se denomina: mercado de un día en adelanto.

El diseño de un mercado de electricidad para energía y servicios auxiliares (SA) con énfasis en la coordinación del despacho económico y reserva rodante se presenta en [1-3]. Dicho modelo permite obtener un mercado de energía eficaz y seguro, que optimiza la programación y el despacho, cumpliendo con los requerimientos de energía y reserva de forma simultánea, además de satisfacer todas las limitaciones de seguridad a menor costo.

En los trabajos reportados en [4-5] se busca establecer el mercado de reserva operacional para determinar la capacidad de reserva óptima y obtener la fijación de precios de reserva en condiciones de equilibrio y los precios de capacidad de reserva, considerándose, a diferencia de la mayoría de las investigaciones, que la capacidad de reserva operacional en un sistema de potencia es flexible.

La formulación y descripción de una metodología para la optimización conjunta de la energía y la reserva considerando la red de transmisión en forma detallada y la confiabilidad del sistema se reporta en [6]. Para la solución del problema se propone el uso de una metodología novedosa de optimización híbrida que integra un algoritmo tradicional de programación no lineal para el cálculo de flujos óptimos de potencia que se encuentra anidado dentro de un algoritmo meta-heurístico conocido como optimización evolutiva por enjambre de partículas (Evolutionary Particle Swarm Optimization, EPSO). EPSO permite realizar el cálculo de la reserva óptima de manera endógena al proceso de optimización, por lo cual la solución obtenida siempre será factible, obteniendo así la mejor solución para el despacho conjunto de energía y reserva.
Otra de las herramientas que recientemente se ha empleado en la industria eléctrica es la Teoría de Opciones Reales [7]. Esta teoría valora aspectos de control de gestión, se inspira básicamente en la teoría de opciones financieras y constituye una de las bases más importantes de la teoría financiera moderna [8]. La teoría de opciones reales permite valorar los activos financieros en condiciones de incertidumbre, a partir de que el precio del activo está en función del valor de otro, que se le denomina subyacente básico [9-10].

La aplicación de la teoría de opciones reales en el sector eléctrico se ha implementado principalmente en la evaluación de proyectos de inversión bajo incertidumbre en los sectores de generación y transmisión [11-18]. Las opciones reales como método de valoración de capacidad de generación de una planta y la política óptima de operación se presentan en [19-20]. En [19] se desarrolla en primer lugar una red de las variables estocásticas y se emplea la programación dinámica hacia atrás para calcular el valor y el funcionamiento óptimo de la política de decisión. En [20] se formula el problema como un problema estocástico multietapas y propone, para la valoración de la central eléctrica, un método de solución que integra la simulación de Monte Carlo y programación dinámica hacia atrás. En el área operativa de los mercados de electricidad, a diferencia que en los sectores de generación y trasmisión, la aplicación de opciones reales ha sido prácticamente nula. Por esto, y al representar un nuevo campo de aplicación, se busca en este trabajo aplicar la teoría de opciones reales utilizándola como metodología de análisis en la toma de decisiones por parte de una GenCo.

El resto del trabajo se estructura de la siguiente manera. La siguiente sección describe la estructura del mercado y los pagos por SA, haciendo énfasis en reservas operativas. Posteriormente se presenta la metodología propuesta. En la sección casos de estudio se muestra con detalle la aplicación de la metodología propuesta y se discuten resultados. Finalmente se presentan las conclusiones del trabajo.

\section{ESTRUCTURA DEL MERCADO Y MECANISMOS DE PAGO PARA LOS SA}

La atención principal de los mercados radica en los mecanismos para la definición del precio del mercado 
y la satisfacción del balance entre proveedores y consumidores. La asignación de unidades en un modelo centralizado, es realizada en función de la potencia activa y la incorporación de reservas rodantes. Sin embargo, esto no es suficiente para que el sistema funcione adecuadamente, se requiere además de otros servicios, denominados SA. Los SA son aquellas funciones que sirven de soporte al proceso de generación, transmisión y distribución de la electricidad, de modo de satisfacer las condiciones de seguridad y calidad [21].

Uno de estos SA es el servicio de Reserva. La reserva en los mercados eléctricos es el producto de mayor importancia para el sistema; su manejo es responsabilidad del operador del sistema (OS) y es quien debe determinar cuánto debe comprar y donde ubicarla. Estas decisiones son determinantes del nivel de confiablidad y la economía alcanzada por el sistema.

Para que exista un mercado de reserva es primordial contar con una estructura adecuada de mercado, en el que exista un marco de seguridad y de calidad en los aspectos tanto económicos como técnicos. Por ello los nuevos mercados tienen como prioridad la transparencia en los cálculos y la búsqueda de los valores óptimos en sus formulaciones. Los nuevos algoritmos a emplear deben obtener una solución justa que maximice el beneficio social y que además sea técnicamente factible y segura.

Aunque existen algunos intentos por uniformar uniformizar las prácticas actuales para la adquisición, localización y tarifación de la reserva, cada mercado ofrece su solución particular. Actualmente existe un amplio consenso en sostener que el servicio de energía y de reserva están fuertemente vinculados, y que deben ser adquiridos simultáneamente mediante la minimización del costo total de producción de energía eléctrica y reserva [1-5, 21-22]. Esta cooptimización debe entonces, asignar simultáneamente las consignas de generación a las distintas unidades y distribuir los requerimientos de reserva entre los agentes del sistema que puedan brindarlo.

Sin embargo, también existe la propuesta que considera que los servicios de energía y reserva están débilmente acoplados. Para esto se utiliza un procedimiento denominado despacho secuencial, en el cual se optimiza en primera instancia el mercado de energía, para luego satisfacer los requerimientos de los distintos tipos de reserva, clasificados según su velocidad de respuesta [21-23]. La propuesta de mercados paralelos funciona en base a subastas horarias, donde las ofertas de ambos servicios se realizan de forma simultánea, pero el equilibrio se realiza en forma secuencial previamente descrito. Este esquema es considerado en el desarrollo de este trabajo de investigación.

En esta interacción se distinguen dos agentes principales: los generadores o productores y el operador del mercado (OM). Los productos a comercializar en los mercados spot, para cada hora, son: i) Energía: suministro de potencia activa (MW), ii) Reserva: la cual debe estar sincronizada con la red (MW).

El pago del servicio de energía se determina en el mercado spot de energía. Respecto a la reserva, el producto que se negocia es sólo la capacidad de suministro. Para las unidades de generación esto sólo representará el costo de oportunidad frente a la producción de energía básica. La utilización real de reservas tiene un costo de producción para los generadores y por consiguiente existe un pago por su uso, el cual es acordado a través del mercado spot de reservas. En el servicio de reserva diferentes alternativas se han propuesto, y en este trabajo las siguientes alternativas son consideradas:

1. Pago único. La reserva es pagada al mismo precio spot de energía establecido en el mercado spot de energía. Independientemente de que la reserva sea solicitada o no por el OS, los proveedores reciben el pago por ésta al precio establecido.

2. Multi-pago: Constituido de un pago por disponibilidad de reserva y un pago por energía. Si la reserva no es requerida, la unidad de generación recibirá únicamente el pago por disponibilidad de reserva establecido en el mercado spot de reserva. Si la reserva es solicitada, se recibe un pago por energía. Este precio de energía es determinado en el mercado de tiempo real.

La operación del mercado y el balance de la energía y la reserva se realizarán en un proceso iterativo, en el cual el OM entregará precios para cada producto en cada hora. Por su parte, 
los generadores ofertan cantidad y precio de cada producto, considerando la maximización de sus beneficios. Esto se relazará en tres etapas secuenciales, despacho inicial ideal, despacho con reserva y despacho final. Es decir la programación de energía del despacho ideal es punto de partida para el despacho con reservas y, así mismo, la siguiente solución es punto de partida para el despacho final. Este proceso durará hasta que se cumpla el equilibrio entre la oferta y la demanda y el cumplimiento de las restricciones de seguridad para la operación del sistema. Sin embargo, en este trabajo solo se considera la determinación de las ofertas enviadas al mercado por parte de la GenCo. El precio del mercado spot, tanto de energía como de reservas, se considera un precio uniforme como se muestra la Figura 1.

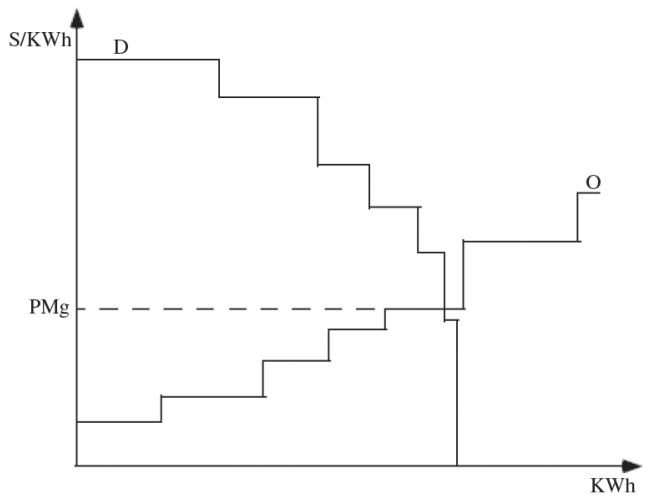

Figura 1. Curvas agregadas de oferta y demanda de energía eléctrica.

Bajo la consideración de que es necesario un redespacho de las unidades debido a requerimientos de red, por ejemplo ante requerimiento de potencia reactiva. Lo anterior implica que generadores en reserva aporten potencia activa al sistema a fin mantener los límites de seguridad dentro de márgenes. Por consecuencia, el precio del mercado (p) se verá afectado, se incrementará. Gráficamente esto se muestra en la Figura 2.

La Figura 2 muestra el incremento en el precio del mercado al incluir el servicio de reserva rodante en un $5 \%$ (p') o en un $10 \%$ (p"). En la misma figura, se muestra que cada unidad participa en la producción del servicio de reserva rodante. En la práctica, no todas las unidades de generación participan en el suministro del servicio de reserva rodante.

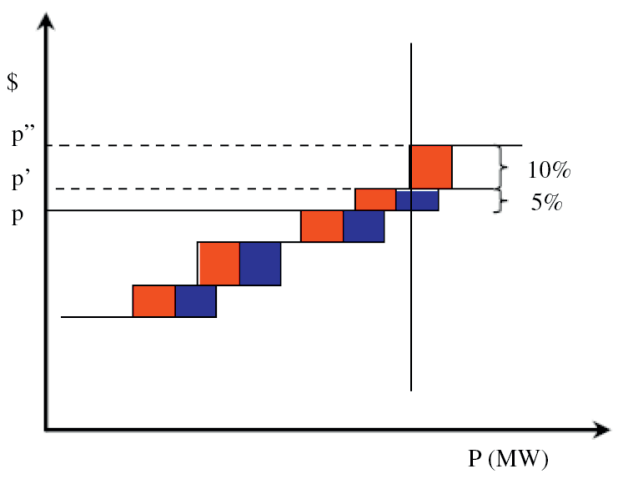

Figura 2. Precios del mercado para energía y reserva.

\section{MODELO PROPUESTO}

La asignación de unidades se ha empleado en la industria eléctrica para determinar las unidades a operar durante un período de tiempo de forma tal de satisfacer la demanda y esto se logre al menor costo. Sin embargo, en los nuevos mercados reestructurados, las GenCos ya no son obligadas a abastecer la demanda [24]. Por lo tanto, las GenCos tienen la libertad de decidir producir o no según las condiciones del mercado.

Dado que las ofertas que las GenCos envían al mercado son compromisos de energía a través de períodos horarios, lo cual se puede ver como opciones estandarizadas ofertadas al mercado en el marco de opciones reales. Los ingresos que perciben por éstas, corresponden, a una combinación de ingresos por concepto de reserva y energía.

La GenCo desea maximizar las ganancias esperadas al ofertar en ambos mercados. Dado que el requerimiento de reserva es impredecible, se simula en forma discreta contingencias en el sistema para evaluar el ejercicio de la opción Americana bajo el mecanismo multi-pago.

\section{Formulación Europea}

Una planta térmica de generación puede describirse como un mecanismo capaz de entregar electricidad en cualquier tiempo donde el precio es igual al costo variable de operación de la planta [25]. Esto corresponde en términos financieros a una opción llamada Europea, que proporciona con esto un soporte al pay-off igual a la diferencia entre el costo actual y el precio establecido de la opción. 


$$
\text { payoff }=p_{E}^{t}-B p_{F}^{t}
$$

donde $p_{E}^{t}$ es el costo actual de la opción, $p_{F}^{t}$ el costo establecido de la opción y $B$ es el coeficiente de producción.

Cuando el uso del servicio es pagado completamente al igual que el no empleado, el programa de optimización es formulado como un conjunto de opciones Europeas. En este caso, 2 subastas son tratadas simultáneamente pero estas pueden ser secuenciales [26]. De esta manera, la ganancia es la suma de las ganancias provenientes de cada subasta. Por lo tanto, la programación de optimización es formulada como un conjunto de opciones Europeas ya que estas son ejercidas hasta el tiempo de madures $t$, donde $t$ representa el período horario de comercio en el mercado spot. La Figura 3 muestra el pay-off para cada servicio en el tiempo $t+1$.

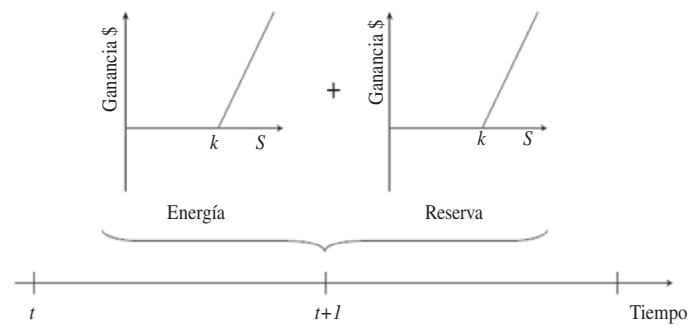

Figura 3. Ofertas simples para cada servicio: PayOff Europeo.

El problema es formulado de la siguiente manera:

$$
\begin{gathered}
\operatorname{Max}\left\{E\left[\left(\sum_{t=1}^{T} \sum_{i=1}^{n} E U\left(R_{t}, C\left(P g_{i, t}\right)-S u-S d\right)\right) u_{i, t}\right]\right\} \\
S . a \sum_{i=1}^{n} P g_{i, t} u_{i, t} \leq D_{t} \quad \forall t=1, \ldots, T \\
\sum_{i=1}^{n} S_{i, t} \leq S_{R, t} \quad \forall t=1, \ldots, T \\
P g_{i, t+1}-P g_{i, t} \leq Z_{i} \quad \forall t=1, \ldots, T \\
P g_{i, t}-P g_{i, t+1} \leq W_{i} \quad \forall t=1, \ldots, T
\end{gathered}
$$

$$
\begin{gathered}
P g_{i, t}^{\mathrm{Min}} \leq P g_{i, t} \leq P g_{i, t}^{\operatorname{Max}} \\
x_{i, t}=\left\{\begin{array}{l}
\min \left(t_{i}^{\text {on }}, x_{i, t+1}+1\right) \text { si } u_{i, t}=1 \\
\max \left(t_{i}^{\text {off }}, x_{i, t+1}-1\right) \text { si } u_{i, t}=0
\end{array}\right. \\
u_{i, t}=\left\{\begin{array}{l}
1 \text { si } 1 \leq x_{i, t-1}<t_{i}^{\text {on }} \\
0 \text { si }-1 \geq x_{i, t-1}>t_{i}^{\text {off }}
\end{array}\right.
\end{gathered}
$$

donde $E$ indica el valor esperado, $E U\left(R_{t}, C\left(P g_{i, t}\right)\right)=\operatorname{Max}\left(P_{t}^{E} P g_{i, t}+P_{t}^{S} S_{i, t}-C\left(P g_{i, t}\right), 0\right)$,

$R_{t}$ es la ganancia económica de la unidad $i$ en el período $t, C\left(P g_{i, t}\right)$ es el costo de operación de la unidad $i$ en período $t, S u$ es el costo de arranque de la unidad $i, S d$ es el costo de paro la unidad $i, P g_{i, t}$ es la potencia de generación activa de la unidad $i$ en el período $t, Z_{i}$ es el incremento máximo en la rampa de subida de la unidad $i, W_{i}$ decremento máximo en la rampa de bajada de la unidad $i, P_{t}^{E}$ es el precio de energía spot en el período $t, P_{t}^{S}$ es el precio de reserva de energía spot en el período $t, D_{t}$ es el requerimiento de potencia activa en el período $t, S_{R, t}$ es la demanda de reserva rodante en el período $t, S_{i, t}$ es la reserva rodante de la unida $i$ en el período $t$, y $n$ es el número de unidades.

La función de costos de la producción de energía por: $C\left(P g_{i, t}\right)=\left\{\begin{array}{cl}P_{t}^{F}\left(a_{i}+b_{i} P g_{i, t}+d_{i} P g_{i, t}^{2}\right) & \text { si } P g_{i, t}>0 \\ 0 & \text { si } P g_{i, t}=0\end{array}\right.$ donde $F \in\{$ Combustóleo, Carbón, Gas Naturalı\}, $a, b$ y $c$ son los parámetros de la curva de costo de la unida de generación $i$.

\section{Formulación Europea-Americana}

Las reservas operativas son requeridas generalmente cuando ocurre una contingencia. Por lo tanto el proceso de tiempo $t$ es externo. La reserva en una opción Americana puede emplearse en cualquier tiempo incluyendo la fecha de madurez. Sin embargo, las ofertas de reserva consisten de una capacidad y precio de energía. Un precio de capacidad describe el valor de pago de capacidad por el operador del sistema para el exacto empleo de la opción a operar. En lo contrario, el precio de energía es pagado únicamente si la opción se ejerce. Para el caso de 
energía se considera la opción Europea. La Figura 4 muestra el pay-off de energía y reserva en el período $t+1$. Los productos consisten de una capacidad y un precio de energía.

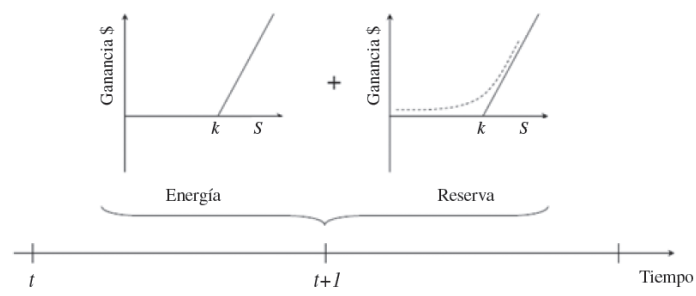

Figura 4. Ofertas simples para cada servicio: PayOff Americano.

El planteamiento del problema bajo la formulación Europeo-Americana se formula a continuación:

$$
\left.\operatorname{Max}\left\{E\left[\left(\begin{array}{l}
\sum_{t=1}^{T} E U\left(R_{t}, C\left(P g_{i, t}\right)\right)+ \\
\sum_{t=1}^{T} A M\left(R_{t}, C_{t}\left(P g_{i, t}\right)\right)-S u-S d
\end{array}\right)\right] u_{i t}\right]\right\}
$$

Sujeto a las restricciones (3)-(9). El primer término en la función objetivo (10) es energía:

$$
E U\left(R_{t}, C\left(P g_{i, t}\right)\right)=\operatorname{Max}\left(P_{t}^{E} P g_{i, t}-C\left(P g_{i, t}\right), 0\right)
$$

El segundo término es la reserva operativa:

$$
\operatorname{Am}\left(R_{t}, C\left(P g_{i, t}\right)\right)=\operatorname{Max}\left(P_{t}^{S} S_{i, t}-C\left(P g_{i, t}\right), 0\right)
$$

Las decisiones operativas en cada período son gráficamente representado en la Figura 5.

En el período $t$, la GenCo podrá decidir si vende reserva, conserva la producción de la cantidad igual de potencia o incrementa/decrementa la producción de potencia. Cualquier decisión es limitada por las restricciones operativas de la unidad de generación.

La probabilidad de ejecutar la opción es una variable externa; esto depende de las condiciones del sistema. Por lo que se asume que el suceso de un evento inesperado tiene la misma probabilidad para cada uno de ellos. Debido a las restricciones operacionales de las unidades la opción puede ser ejecutada únicamente en cierto número de tiempos dentro del período planeado.

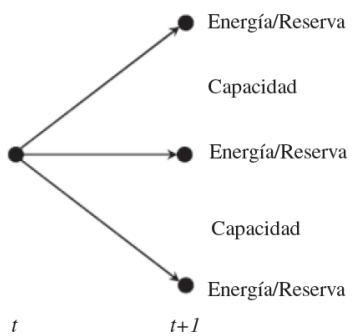

Figura 5. Decisión operativa en el periodo $t+1$.

A continuación, se presenta varios ejemplos numéricos. Las simulaciones de optimización fueron desarrolladas en el paquete computacional MATLAB®.

\section{CASOS DE ESTUDIO}

Tres casos de estudio se reportan en este artículo derivados de un caso base en donde una GenCo, constituida de cuatro unidades generadoras, participa en los mercado spot de energía y reserva. En muchos sistemas la reserva se asigna como un porcentaje de la demanda (4-6\%), lo cual se asume en este trabajo. Las unidades de generación de la compañía se encuentran ubicadas dentro de una misma central generadora, lo cual se representa en la Figura 6.

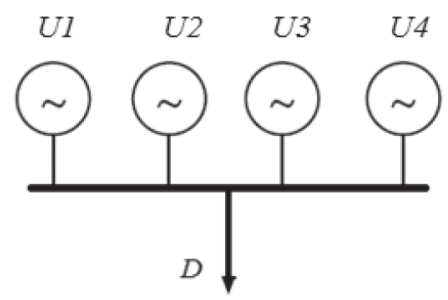

Figura 6. Unidades de generación de la GenCo.

La Tabla 1 presenta las características de las unidades y el patrón de la demanda residual se muestra en la Tabla 2.

El primer caso corresponde al caso base, en donde se supone que GenCo participa únicamente en el mercado spot de energía. La GenCo participa además en el mercado spot de reserva en el segundo y tercer caso de estudio. 
Tabla 1. Parámetros de la curva de costo de producción y límites operativos de las unidades de generación.

\begin{tabular}{|c|c|c|c|c|c|}
\hline Unidad & $\begin{array}{c}\mathbf{a} \\
(\$)\end{array}$ & $\begin{array}{c}\mathbf{b} \\
(\$ / \mathbf{M W})\end{array}$ & $\begin{array}{c}c \\
\left(\$ / \mathbf{M W}^{2}\right)\end{array}$ & $\begin{array}{l}P g_{i}^{\operatorname{Min}} \\
(\mathbf{M W})\end{array}$ & $\begin{array}{l}P g_{i}^{\operatorname{Max}} \\
\text { (MW) }\end{array}$ \\
\hline 1 & 213.00 & 7.69750 & 0.001 & 25 & 80 \\
\hline 2 & 585.62 & 6.40752 & 0.001 & 60 & 250 \\
\hline 3 & 684.74 & 6.14753 & 0.001 & 75 & 300 \\
\hline 4 & 252.00 & 7.64000 & 0.001 & 20 & 60 \\
\hline
\end{tabular}

Tabla 2. Patrón de demanda residual.

\begin{tabular}{|c|c|c|c|}
\hline Hora & Carga (MW) & Hora & Carga (MW) \\
\hline 1 & 450 & 5 & 400 \\
\hline 2 & 530 & 6 & 280 \\
\hline 3 & 600 & 7 & 290 \\
\hline 4 & 540 & 8 & 500 \\
\hline
\end{tabular}

El caso dos consiste en suponer que GenCo al participar en el mercado de energía y reserva recibe un pago único por cada servicio. En el caso de la reserva, el pago por disponibilidad será el mismo que el de suministro. Los precios por cada servicio, se establecen en el mercado spot correspondiente.

El último caso de estudio es similar al caso dos, diferirá de él únicamente en la forma de pago para el servicio de reserva. En el caso dos, se considera que se recibe por él un pago único, en cambio en el tercer caso, se recibe por éste una retribución constituida por dos componentes de pago, un pago por disponibilidad de reserva y otro por energía (precio de la reserva al ser solicitada en tiempo real).

Para el análisis de los casos, por simplicidad y debido a que las rampas de subida o bajada de potencia limitan la potencia entregada en un período según la potencia producida en el período anterior, no se toman en consideración.

\section{Caso 1}

Se realiza la asignación óptima de unidades de generación suponiendo que la GenCo únicamente oferta energía, con el propósito de comparar, posteriormente, los resultados que se obtuvieron al considerar también un mercado de reserva.
La asignación óptima se muestra en la Tabla 3.

De la misma Tabla 3 se observa que la unidad 3, por ser la más económica, siempre participa en su límite máximo o muy cercano a él. En cambio, por ser las unidades de generación más costosas, la unidad 4 nunca entra al despacho y la unidad 1 solo participa en el período tres, que es el de mayor demanda. En este caso la unidad 2 será, para la mayoría de los casos, la unidad marginal del conjunto de unidades de la GenCo.

Tabla 3. Asignación optima de unidades considerando únicamente participación en el mercado de energía.

\begin{tabular}{|c|c|c|c|c|}
\hline \multirow{2}{*}{ Hora } & \multicolumn{4}{|c|}{ Energía (MW) } \\
\cline { 2 - 5 } & U1 & U2 & U3 & U4 \\
\hline 1 & 0 & 160.0025 & 289.9975 & 0 \\
\hline 2 & 0 & 230 & 300 & 0 \\
\hline 3 & 50 & 250 & 300 & 0 \\
\hline 4 & 0 & 240 & 300 & 0 \\
\hline 5 & 0 & 135.0025 & 264.9975 & 0 \\
\hline 6 & 0 & 0 & 280 & 0 \\
\hline 7 & 0 & 0 & 290 & 0 \\
\hline 8 & 0 & 200 & 300 & 0 \\
\hline
\end{tabular}

En la Tabla 4 se muestran los costos de producción de cada unidad de generación por período, al igual que el costo total de producción de la GenCo. En la misma tabla, se aprecia que los costos fijos de las unidades de generación 1 y 4, \$213 y \$252 respectivamente, se acumulan en cada período cuando estas están en estado de apagado.

Tabla 4. Costos de producción variables y totales (\$) de cada unidad de generación por periodo.

\begin{tabular}{|c|c|c|c|c|c|}
\hline Hora & U1 & U2 & U3 & U4 & Total \\
\hline 1 & 213.00 & 1636.45 & 2551.61 & 252 & 4653.05 \\
\hline 2 & 213.00 & 2112.26 & 2619.00 & 252 & 5196.26 \\
\hline 3 & 600.38 & 2250.01 & 2619.00 & 252 & 5721.38 \\
\hline 4 & 213.00 & 2181.03 & 2619.00 & 252 & 5265.03 \\
\hline 5 & 213.00 & 1468.88 & 2384.04 & 252 & 4317.93 \\
\hline 6 & 213.00 & 585.62 & 2484.45 & 252 & 3535.07 \\
\hline 7 & 213.00 & 585.62 & 2551.62 & 252 & 3602.24 \\
\hline 8 & 213.00 & 1907.13 & 2619.00 & 252 & 4991.13 \\
\hline
\end{tabular}


En la Tabla 5 se muestra el precio de energía y las ganancias que se obtienen por cada una de las unidades generadoras por período. También se indican las ganancias totales que obtuvo GenCo en cada período.

Tabla 5. Precio de energía y ganancias de cada unidad de generación y totales por periodo.

\begin{tabular}{|c|c|c|c|c|c|c|}
\hline Hora & $\begin{array}{c}\text { Precio de } \\
\text { Energía } \\
\mathbf{( \$ / M W h ) ~}\end{array}$ & $\begin{array}{c}\mathbf{U 1} \\
\mathbf{( \$ )}\end{array}$ & $\begin{array}{c}\mathbf{U 2} \\
\mathbf{( \$ )}\end{array}$ & $\begin{array}{c}\mathbf{U 3} \\
\mathbf{( \$ )}\end{array}$ & $\begin{array}{c}\mathbf{U 4} \\
\mathbf{( \$ )}\end{array}$ & $\begin{array}{c}\text { Totales } \\
\mathbf{( \$ )}\end{array}$ \\
\hline 1 & 11.64 & -213.00 & 225.98 & 823.96 & -252 & 584.95 \\
\hline 2 & 11.24 & -213.00 & 472.94 & 753.00 & -252 & 760.94 \\
\hline 3 & 11.46 & -27.38 & 614.99 & 819.00 & -252 & 1154.62 \\
\hline 4 & 11.20 & -213.00 & 506.97 & 741.00 & -252 & 782.97 \\
\hline 5 & 11.97 & -213.00 & 147.10 & 787.98 & -252 & 470.07 \\
\hline 6 & 11.18 & -213.00 & -585.62 & 645.95 & -252 & -404.67 \\
\hline 7 & 11.09 & -213.00 & -585.62 & 664.48 & -252 & -386.14 \\
\hline 8 & 11.38 & -213.00 & 368.87 & 795.00 & -252 & 698.87 \\
\hline
\end{tabular}

Se puede observar de la Tabla 5 que las unidades de generación que tuvieron poca participación en el mercado obtendrán pérdidas y que las unidades más económicas (unidades 2 y 3 ) son las que obtienen los mayores beneficios.

Respecto a las resultados totales que se muestran en la Tabla 5, se puede observa que la GenCo obtendrá ganancias en la mayoría de los períodos, aun y cuando algunas de sus unidades reporte pérdidas debido a no participar en el despacho. En estos casos, la GenCo solventa los costos fijos de producción con las unidades que sí están en funcionamiento.

Si en un determinado momento se estableciera como estrategia de la GenCo para cada período la participación de todos los generadores, fijando a un determinado nivel de potencia las unidades más caras (unidades de generación 1 y 4), las ganancias totales obtenidas por GenCo serían prácticamente las mismas, pues aunque se reducirían las pérdidas de dichas unidades, las ganancias obtenidas por la unidades 2 y 3 también reducirían, ya que la potencia suministrada por éstas sería menor dada la participación de las otras dos unidades de generación.

\section{Caso 2}

A continuación se integra el mercado spot de reservas al mercado spot de energía. El requerimiento de reserva en cada período de tiempo se considera igual al 10\% de la demanda residual esperada.

El esquema de pago para este caso de estudio está dado bajo una formulación Europea a través de un pago único para cada uno de los servicios. Para el servicio de reserva se considera que el precio de reserva será igual al precio de energía, sin importar si la reserva es requerida o no por el OS. Es decir, el pago por reserva suministrada será igual al pago por disponibilidad de reserva. Los resultados que se obtuvieron se muestran en las Tablas 6-9.

En la Tabla 6 se presentan los requerimientos de energía y reserva para cada período. La asignación óptima de energía, indicando que unidades se encuentran en línea y con cuanta potencia participan, se reporta también en la Tabla 6. De igual forma, en esta tabla se muestra la participación de cada unidad en el mercado de reserva.

De la Tabla 6 se puede observar que la unidad 3 sigue ofertando a su capacidad máxima, teniendo la mayor participación tanto en el mercado de energía como en el de reserva. La unidad 2 dejará de ser la unidad marginal de la GenCo en energía, sin embargo, lo será para el servicio de reserva. El precio del servicio de reserva será establecido en casi todos los períodos por esta unidad. La unidad 1 tiene una participación mayor en el mercado de energía, respecto al caso anterior, debido al requerimiento de reserva. En cuanto al mercado de reserva esta unidad tendrá poca participación. La unidad 4 sigue teniendo una escasa participación en ambos mercados, sin embargo, el hecho de participar en la demanda pico y ser considerada en el mercado de reserva, le permite tener menores pérdidas, como se aprecia en la Tabla 9.

Los precios por los servicios de energía y reserva, así como los costos de producción de cada unidad por período y el costo total del mismo se muestran en la Tabla 7. Los costos de producción mostrados en esta tabla cambian respecto a los que se reportan en la Tabla 4, debido a que la participación en el mercado de energía por parte de las unidades generadoras no es la misma dada la solicitud de potencia para el servicio de reserva.

Es importante menciona que los costos de producción de las unidades participantes en ambos mercados 
Tabla 6. Asignación optima de unidades considerando despacho simultáneo de energía y reservas.

\begin{tabular}{|c|c|c|c|c|c|c|c|c|c|c|}
\hline \multirow{2}{*}{ Hora } & \multirow{2}{*}{$\begin{array}{c}\text { Demanda } \\
\text { (MW) }\end{array}$} & Reserva & \multicolumn{4}{|c|}{ Energía (MW) } & \multicolumn{4}{|c|}{ Reserva (MW) } \\
\cline { 6 - 13 } & & U1 & U2 & U3 & U4 & U1 & U2 & U3 & U4 \\
\hline 1 & 450 & 45 & 0 & 180 & 270 & 0 & 0 & 15 & 30 & 0 \\
\hline 2 & 530 & 53 & 35 & 225 & 270 & 0 & 0 & 23 & 30 & 0 \\
\hline 3 & 600 & 60 & 51 & 225 & 270 & 54 & 0 & 25 & 30 & 5 \\
\hline 4 & 540 & 54 & 45 & 225 & 270 & 0 & 0 & 24 & 30 & 0 \\
\hline 5 & 400 & 40 & 0 & 135.0025 & 264.9975 & 0 & 4 & 0 & 30 & 6 \\
\hline 6 & 280 & 28 & 55 & 225 & 0 & 0 & 3 & 25 & 0 & 0 \\
\hline 7 & 290 & 29 & 25 & 0 & 265 & 0 & 4 & 25 & 0 & 0 \\
\hline 8 & 500 & 50 & 25 & 205 & 270 & 0 & 0 & 20 & 30 & 0 \\
\hline
\end{tabular}

Tabla 7. Costos de producción variables y totales (\$) de cada unidad de generación por periodo.

\begin{tabular}{|c|c|c|c|c|c|c|c|}
\hline \multirow{2}{*}{ Hora } & \multirow{2}{*}{$\begin{array}{c}\text { Precio } \\
\text { Energía (\$/ } \\
\text { MWh) }\end{array}$} & \multirow{2}{*}{$\begin{array}{c}\text { Precio } \\
\text { Reserva (\$/ } \\
\text { MWh) }\end{array}$} & \multicolumn{4}{|c|}{ Costos (\$) } & \multirow{2}{*}{$\begin{array}{c}\text { Costo Total } \\
\text { (\$) }\end{array}$} \\
\hline & & & U1 & U2 & $\mathbf{U 3}$ & $\mathrm{U} 4$ & \\
\hline 1 & 11.666 & 11.666 & 213.00 & 1873.12 & 2619.00 & 252.00 & 4957.12 \\
\hline 2 & 11.730 & 11.730 & 483.64 & 2236.20 & 2619.00 & 252.00 & 5590.84 \\
\hline 3 & 12.630 & 12.630 & 608.17 & 2250.01 & 2619.00 & 706.24 & 6183.42 \\
\hline 4 & 11.730 & 11.730 & 561.41 & 2243.10 & 2619.00 & 252.00 & 5675.51 \\
\hline 5 & 11.990 & 11.990 & 243.81 & 1468.88 & 2585.27 & 297.88 & 4595.83 \\
\hline 6 & 12.135 & 12.135 & 662.82 & 2250.01 & 684.74 & 252.00 & 3849.57 \\
\hline 7 & 11.973 & 11.973 & 437.07 & 746.43 & 2384.06 & 252.00 & 3819.56 \\
\hline 8 & 11.890 & 11.890 & 406.06 & 2077.95 & 2619.00 & 252.00 & 5355.01 \\
\hline
\end{tabular}

incluyen los costos relacionados a la transformación de la reserva en energía, asumiendo con ello la condición de entrega de reserva al sistema. Si la reserva no se requiere por el sistema, las ganancias serán mayores dado que los costos de producción serán menores.

Los ingresos obtenidos por las unidades de generación al participar en los mercados de energía y reserva se muestran en la Tabla 8. Las ganancias resultantes para cada una de ellas se muestran en la Tabla 9. En este caso cada una de las unidades recibirá mayores ganancias, porque al recibir el pago por disponibilidad de reserva, sumada a los ingresos obtenidos por la venta de energía, hace posible compensar los costos de producción.

\section{Caso 3}

Para el siguiente análisis, y para facilidad de explicación del ejercicio de la Opción Americana, únicamente se considera a la unidad de generación número 3 y el período de máxima demanda (período tres). Para lo cual, primeramente se analiza el mismo período de acuerdo a las condiciones del caso 2 . Se supe que al término del tiempo de ejercicio el OS no requiere la energía reservada por el generador, sin embargo, dicho generador seguirá recibiendo el pago por reserva, ya que se le pagará por haber estado disponible. Los resultados se muestran en la Tabla 10.

De la Tabla 10 se observa que el generador obtendrá mayores ganancias cuando el OS no le requiera la reserva. Lo anterior debido a que únicamente verá reflejados los costos de producir 270MW en el período, además de recibir el pago por reserva al haber estado disponible.

La formulación Americana se aplicará en la retribución del servicio de reserva. Mientras la 
Tabla 8. Ingresos obtenidos al participar en ambos mercados: energía y reservas.

\begin{tabular}{|c|r|r|r|r|r|r|r|r|}
\hline \multirow{2}{*}{ Hora } & \multicolumn{4}{|c|}{ Ingresos Energía (\$) } & \multicolumn{4}{c|}{ Ingresos Reserva (\$) } \\
\cline { 2 - 9 } & \multicolumn{1}{c|}{ U1 } & \multicolumn{1}{c|}{ U2 } & \multicolumn{1}{c|}{ U3 } & \multicolumn{1}{c|}{ U4 } & U1 & \multicolumn{1}{c|}{ U2 } & \multicolumn{1}{c|}{ U3 } & \multicolumn{1}{c|}{ U4 } \\
\hline 1 & 0.00 & 2099.88 & 3149.82 & 0.00 & 0.00 & 174.99 & 349.98 & 0.00 \\
\hline 2 & 410.55 & 2639.25 & 3167.10 & 0.00 & 0.00 & 269.79 & 351.90 & 0.00 \\
\hline 3 & 644.13 & 2841.75 & 3410.10 & 682.02 & 0.00 & 315.75 & 378.90 & 63.15 \\
\hline 4 & 527.85 & 2639.25 & 3167.10 & 0.00 & 0.00 & 281.52 & 351.90 & 0.00 \\
\hline 5 & 0.00 & 1618.68 & 3177.32 & 0.00 & 47.96 & 0.00 & 359.70 & 71.94 \\
\hline 6 & 667.43 & 2730.40 & 0.00 & 0.00 & 36.41 & 303.38 & 0.00 & 0.00 \\
\hline 7 & 299.33 & 0.00 & 3172.87 & 0.00 & 47.89 & 299.33 & 0.00 & 0.00 \\
\hline 8 & 297.25 & 2437.45 & 3210.30 & 0.00 & 0.00 & 237.80 & 356.70 & 0.00 \\
\hline
\end{tabular}

Tabla 9. Ganancias (\$) de cada unidad de generación $\mathrm{y}$ totales por periodo.

\begin{tabular}{|c|r|r|r|r|r|}
\hline Hora & \multicolumn{1}{c|}{ U1 } & U2 & \multicolumn{1}{c|}{ U3 } & U4 & Totales \\
\hline 1 & -213.00 & 401.75 & 880.80 & -252.00 & 817.55 \\
\hline 2 & -73.09 & 672.84 & 900.00 & -252.00 & 1247.75 \\
\hline 3 & 35.96 & 907.49 & 1170.00 & 38.93 & 2152.38 \\
\hline 4 & -33.56 & 677.67 & 900.00 & -252.00 & 1292.11 \\
\hline 5 & -195.85 & 149.80 & 951.75 & -225.94 & 679.77 \\
\hline 6 & 41.02 & 783.77 & -684.74 & -252.00 & -111.96 \\
\hline 7 & -89.85 & -447.11 & 788.81 & -252.00 & -0.14 \\
\hline 8 & -108.81 & 597.30 & 948.00 & -252.00 & 1184.49 \\
\hline
\end{tabular}

Tabla 10. Ganancias obtenidas por la unidad de generación 3.

\begin{tabular}{|c|c|c|c|}
\hline $\begin{array}{c}\text { Precio } \\
\text { Energía } \\
\text { (\$/MWh) }\end{array}$ & $\begin{array}{c}\text { Precio de } \\
\text { Reserva } \\
\text { (\$/MWh) }\end{array}$ & $\begin{array}{c}\text { Ganancias } \\
\text { al ejercer } \\
\text { la opción } \\
(\$)\end{array}$ & $\begin{array}{c}\text { Ganancias } \\
\text { al no } \\
\text { ejercer la } \\
\text { opción (\$) }\end{array}$ \\
\hline 12.630 & 12.630 & 1170.00 & 1371.53 \\
\hline
\end{tabular}

reserva no sea requerida, se recibirá un pago por disponibilidad de reserva. Cuando la reserva sea requerida y se ejerza la opción, se recibirá un pago por energía.

Para evaluar la opción Americana de la formulación Europea-Americana, en el presente caso de estudio, se ejerce la opción mediante la simulación de una contingencia acontecida entre cada uno de los períodos, lo cual hace necesario el uso de la reserva para cumplir con los requerimientos de demanda.
Para fines explicativos se simula el ejercicio de la Opción en forma discreta (minuto 15, 30 y 45 después de iniciado el período) mediante la simulación de contingencia y por consecuencia el uso de la reserva ante la nueva condición operativa.

Debido a que el período de tiempo en el que se trabaja es de una hora y que la contingencia ocurrirá en un determinado instante de ésta, es necesario que las curvas de costos de las unidades de generación sean dadas en minutos. Las curvas bajo estas condiciones son:

$$
C\left(P g_{1}\right)=3,55+0,12829 P g_{1}+0,000017 P g_{1}^{2} \frac{\$}{\min }
$$$$
C\left(P g_{2}\right)=9,76+0,10679 P g_{2}+0,000017 P g_{2}^{2} \frac{\$}{\min }
$$$$
C\left(P g_{3}\right)=11,41+0,10245 P g_{3}+0,000017 P g_{3}^{2} \frac{\$}{\min }
$$$$
C\left(P g_{4}\right)=4,2+0,12733 P g_{4}+0,000017 P g_{4}^{2} \frac{\$}{\min }
$$

Suponiendo que al minuto 15,30 ó 45 después de iniciado el período 3 la demanda se incrementa en $60 \mathrm{MW}$, lo cual implica que la demanda en tiempo real es de 660MW. La unidad 3 opera a potencia máxima dado que la reserva se transforma en energía al ser despachada una vez ocurrida la contingencia.

El precio de reserva antes de suscitarse la contingencia es la prima por disponibilidad. Ocurrida la contingencia, la opción Americana es ejercida y el precio de la opción es el precio en tiempo real de energía desde el momento en que la reserva es requerida y hasta el final del período. 
Los precios para cada uno de los servicios proporcionados por la unidad de generación 3 que se emplean en la solución de este caso se muestran en la Tabla 11. Los precios de energía en tiempo real se calculan en el instante en que se presenta el incremento de demanda.

Tabla 11. Precios para los servicios de energía y reserva.

\begin{tabular}{|c|c|c|c|}
\hline $\begin{array}{c}\text { Tiempo de } \\
\text { inicio de la } \\
\text { contingencia } \\
(\mathrm{min})\end{array}$ & $\begin{array}{c}\text { Precio de } \\
\text { Energía } \\
\text { Spot } \\
(\$ / \text { MW min) }\end{array}$ & $\begin{array}{c}\text { Precio de } \\
\text { Disponibilidad } \\
\text { de Reserva } \\
\text { (\$/MW min) }\end{array}$ & $\begin{array}{c}\text { Precio de } \\
\text { Energía en } \\
\text { tiempo real } \\
\text { (\$/MW min) }\end{array}$ \\
\hline 15 & 3.158 & 0.570 & 3.171 \\
\hline 30 & 6.315 & 1.141 & 6.343 \\
\hline 45 & 9.473 & 1.711 & 9.514 \\
\hline
\end{tabular}

Los resultados que se obtienen para el caso de estudio se muestran en las Tablas 12-14. De la Tabla 12 se observan los costos y ganancias que se obtuvieron para el intervalo en el que el generador estuvo operando en condiciones normales, es decir, suministrando $270 \mathrm{MW}$ de energía y teniendo 30MW disponibles como reserva. Se observan también, los costos y ganancias al considerarse que la unidad opera a potencia máxima al ocurrir la contingencia (la reserva se considera ahora energía). Los ingresos que obtiene el generador por proporcionar los servicios de energía y reserva en cada intervalo (precontingencia y postcontingencia) se reportan en la Tabla 13.

Los resultados que se reportan en las Tablas 12 y 13 hacen posible observar los cambios que ocurren en los ingresos, costos de producción y ganancias al presentarse la contingencia y al considerarse el esquema multi-pago para reserva.

Así por ejemplo, al situarse en el caso en que la contingencia inicia al minuto 15 del período, se observa en la Tabla 12, en su primera fila la potencia de participación del generador, la potencia destinada a energía, la potencia asignada como reserva, los precios de energía y disponibilidad de reserva, los costos de producción y las ganancias; todos estos relacionados a la operación de la unidad en condiciones normales durante los primeros 15 minutos. Es decir, en este período el generador proporcionará $270 \mathrm{MW}$ como energía a un precio de 3.158 \$/MW, teniendo además 30MW disponibles como reserva, los cuales tienen un precio de 0.570 \$/MW. Los ingresos por estos pagos se muestran en la tercera fila de la Tabla 13.

Los costos de producción que se muestran en la primera fila de la Tabla 12 y que se han calculado hasta este momento, solo reflejarán los gastos en que incurre la unidad al generar durante los primeros 15 minutos 270MW. Las ganancias que se muestran en ésta, son los ingresos obtenidos por el generador al participar en los mercados de energía y reserva menos los costos de producción correspondientes.

En la segunda fila de la Tabla 12 se muestran los mismos datos que se mencionaron anteriormente, pero ahora la reserva se ha convertido en energía y se tiene un precio de energía calculado en tiempo real para realizar su pago. Así, el generador recibirá un pago de energía igual a 9.473 \$/MW

Tabla 12. Resultados obtenidos para la unidad 3 una vez ocurrida la contingencia.

\begin{tabular}{|c|c|c|c|c|c|c|c|c|c|}
\hline $\begin{array}{c}\text { Tiempo de } \\
\text { inicio de la } \\
\text { contingencia } \\
\quad(\min )\end{array}$ & Minuto & $\begin{array}{c}\text { Potencia } \\
\text { (MW) }\end{array}$ & $\begin{array}{c}\text { Costo } \\
(\$)\end{array}$ & $\begin{array}{c}\text { Energía } \\
\text { (MW) }\end{array}$ & $\begin{array}{c}\text { Reserva } \\
\text { (MW) }\end{array}$ & $\begin{array}{c}\text { Precio } \\
\text { Energía } \\
\text { Spot } \\
(\$ / M W \\
\text { min) }\end{array}$ & $\begin{array}{c}\text { Precio } \\
\text { Reserva } \\
\text { (\$/MW } \\
\text { min) }\end{array}$ & \begin{tabular}{|c} 
Precio \\
Energía \\
en \\
Tiempo \\
Real (\$/ \\
MW min)
\end{tabular} & $\begin{array}{c}\text { Ganancia } \\
(\$)\end{array}$ \\
\hline \multirow{2}{*}{15} & 15 & 270 & 604.73 & 270 & 30 & 3.158 & 0.570 & --- & 264.91 \\
\hline & 45 & 300 & 1965.60 & 300 & 0 & 9.473 & --- & 3.171 & 687.12 \\
\hline \multirow{2}{*}{30} & 30 & 270 & 1209.47 & 270 & 30 & 6.315 & 1.141 & --- & 529.81 \\
\hline & 30 & 300 & 1310.40 & 300 & 0 & 6.315 & --- & 6.343 & 584.94 \\
\hline \multirow{2}{*}{45} & 45 & 270 & 1814.20 & 270 & 30 & 9.473 & 1.711 & --- & 794.72 \\
\hline & 15 & 300 & 655.20 & 300 & 0 & 3.158 & --- & 9.514 & 482.76 \\
\hline
\end{tabular}


Tabla 13. Ingresos obtenidos al participar en los mercados de energía y reserva.

\begin{tabular}{|c|c|c|c|c|}
\hline $\begin{array}{c}\text { Tiempo de } \\
\text { inicio de la } \\
\text { contingencia } \\
\text { (min) }\end{array}$ & Minuto & $\begin{array}{c}\text { Ingresos } \\
\text { por } \\
\text { Energía } \\
\mathbf{( \$ )}\end{array}$ & $\begin{array}{c}\text { Ingresos } \\
\text { por } \\
\text { Reserva } \\
\mathbf{( \$ )}\end{array}$ & $\begin{array}{c}\text { Ingresos } \\
\text { por ambos } \\
\text { servicios } \\
\mathbf{\$ \$}\end{array}$ \\
\hline \multirow{2}{*}{15} & 15 & 852.53 & 17.11 & 869.64 \\
\cline { 2 - 5 } & 45 & 2557.58 & 95.14 & 2652.72 \\
\hline \multirow{2}{*}{30} & 30 & 1705.05 & 34.23 & 1739.28 \\
\cline { 2 - 5 } & 30 & 1705.05 & 190.29 & 1895.34 \\
\hline \multirow{2}{*}{45} & 45 & 2557.58 & 51.34 & 2608.92 \\
\cline { 2 - 5 } & 15 & 852.53 & 285.43 & 1137.96 \\
\hline
\end{tabular}

por los 270MW comprometidos desde un inicio (opción Europea) y un pago de energía calculado en tiempo real igual a $3.171 \$ / \mathrm{MW}$ por los $30 \mathrm{MW}$ de reserva que ahora se han convertido en energía (opción Americana). Los ingresos que se obtienen por lo anterior se presentan en la segunda fila de la Tabla 13. Los costos que se muestran en la Tabla 12 para este caso, son los que obtiene el generador al operar durante 45 minutos a 300MW debido a las nuevas condiciones del sistema.

Es importante mencionar, que durante el período estudiado y para los casos que se muestran en esta sección, el generador 3 vende siempre $300 \mathrm{MW}$, solo que antes del minuto 15,30 ó 45 vende energía y reserva, después de estos (al simularse la contingencia) solo vende energía. Sin embargo para este último caso habrá pagos diferentes para cada bloque de energía, uno será retribuido a un precio establecido por el mercado de 24 horas en adelanto y el otro se pagará a un precio resultado de la operación en tiempo real.

La Tabla 14 contiene los resultados totales de los costos de producción y las ganancias que obtuvo la unidad de generación en el período 3. De ella se observa que los costos de producción serán menores conforme la duración de la contingencia sea menor, puesto que el generador trabajará a potencia máxima menor tiempo. Las ganancias que obtendrá se incrementarán cuando la contingencia ocurra más cercana al término del período, es decir, cuando su duración sea menor. Esto debido a que los costos de producción serán menores y a que el precio de energía en tiempo real será mayor.
Tabla 14. Costos y ganancias totales obtenidas en el periodo.

\begin{tabular}{|c|c|c|}
\hline $\begin{array}{c}\text { Tiempo de inicio } \\
\text { de la contingencia } \\
\text { (min) }\end{array}$ & Costos $\mathbf{( \$ )}$ & Ganancia (\$) \\
\hline 15 & 2570.33 & 952.03 \\
\hline 30 & 2519.87 & 1114.75 \\
\hline 45 & 2469.40 & 1277.48 \\
\hline
\end{tabular}

Se considera ahora que al término del tiempo de ejercicio (60 minutos) el OS no solicita al generador su reserva. Ante esto, el generador tendrá únicamente como pago el precio por disponibilidad de reserva. Los resultados se muestran en la Tabla 15. De esta tabla se observa que es mucho más conveniente para el generador que el OS requiera su reserva. Aunque si éste decidiera no hacerlo, no significaría para el generador obtener pérdidas en el período, únicamente obtendría menores ganancias.

Tabla 15. Ganancias obtenidas por la unidad de generación 3.

\begin{tabular}{|c|r|r|}
\hline $\begin{array}{c}\text { Inicio de } \\
\text { contingencia }\end{array}$ & $\begin{array}{c}\text { Ganancias al } \\
\text { ejercer la opción }\end{array}$ & $\begin{array}{c}\text { Ganancias al no } \\
\text { ejercer la opción }\end{array}$ \\
\hline 15 & 952.03 & 1061.09 \\
\hline 30 & 114.75 & 1061.09 \\
\hline 45 & 1277.48 & 1061.09 \\
\hline
\end{tabular}

Los resultados que se muestran a continuación son los obtenidos para la unidad de generación 3 en el período de máxima demanda (período 3) para cada uno de los distintos casos de estudio. Dichos resultados se muestran en la Tabla 16.

Tabla 16. Costos y ganancias totales obtenidos en el periodo.

\begin{tabular}{|c|c|c|c|c|}
\hline Caso & $\begin{array}{c}\text { Inicio de } \\
\text { contingencia } \\
(\mathrm{min})\end{array}$ & $\begin{array}{c}\text { Costos } \\
(\$)\end{array}$ & $\begin{array}{c}\text { Ganancias } \\
\text { al ejercer } \\
\text { la opción } \\
(\$)\end{array}$ & $\begin{array}{c}\text { Ganancias al } \\
\text { no ejercer la } \\
\text { opción (\$) }\end{array}$ \\
\hline Base & -- & 2619.00 & 819.00 & -- \\
\hline $\begin{array}{l}\text { Pago } \\
\text { único }\end{array}$ & --- & 2619.00 & 1170.00 & 1371.53 \\
\hline \multirow{4}{*}{$\begin{array}{c}\text { Multi- } \\
\text { pago }\end{array}$} & 15 & 2570.33 & 952.03 & \multirow{4}{*}{1061.09} \\
\hline & 30 & 2519.87 & 1114.75 & \\
\hline & 45 & 2469.40 & 1277.48 & \\
\hline & 59 & 2422.30 & 1429.36 & \\
\hline
\end{tabular}


Al comparar los resultados de la Tabla 16 se puede decir que:

- Es más conveniente para la unidad de generación 3 participar en el mercado de energía y reserva, que únicamente en el mercado de energía. Aunque en ambos casos el generador participa a potencia máxima, el hecho de aperar a 270MW (hasta que la reserva sea requerida) y recibir el pago por disponibilidad de reserva (30MW) le permite obtener ganancias mayores.

- Los costos de producción son menores en la formulación Europeo-Americana (esquema multi-pago para el servicio de reserva), debido a que la unidad de generación no trabaja los 60 minutos a potencia máxima. Por ejemplo al considerar el requerimiento de reserva al minuto 45 , los costos de producción serán la suma de producir durante 45 minutos $270 \mathrm{MW}$ más los costos de producir 15min 300MW.

- Considerándose la posibilidad de que el OS no requiera la potencia de reserva, es decir, que únicamente se reciba el pago por disponibilidad, sería económicamente más conveniente participar en el mercado de reserva bajo la formulación Europea (esquema de pago único). Sin embargo como esto es impredecible, resulta más redituable participar en este mercado bajo un esquema multi-pago (formulación Americana).

- El hecho de que el OS no ejerce la opción, es decir, que no requiera la reserva, no debe implicar pérdidas para la unidad de generación. Al final del período las ganancias serán menores a las que se pudieran obtener si la reserva hubiera sido solicitada.

- Considerando tanto la maximización de ganancias como la posibilidad de que la opción no se ejerza, es definitivamente recomendable que la unidad de generación 3 participe en el mercado de energía y reserva bajo el esquema de pago formulado como una opción Europea (pago único para cada servicio).

- Si las condiciones del mercado y la reglamentación de éste lo permite, una unidad de generación que no ha tenido participación importante en el despacho de energía por ser una unidad cara, puede mejorar su situación si al presentarse una contingencia le es requerida una aportación adicional de potencia y decide vender su capacidad disponible a un precio igual (como mínimo) al precio de energía pactado.

\section{CONCLUSIONES}

Con la reestructuración del sector eléctrico y la desagregación de las diferentes actividades se vislumbra la posibilidad de que cada uno de los servicios involucrados en el suministro de energía eléctrica sean tratados en mercados independientes. En este trabajo se ha considerado la existencia de dos mercados paralelos: Energía y Reserva. De los resultados presentados se concluye lo siguiente.

Las condiciones de pago tanto para energía como para reserva, deberán estar debidamente establecidas en las reglas del mercado y bajo éstas, la GenCo buscará la decisión que maximice sus ganancias en el corto plazo, y eventualmente en el largo plazo. Si las condiciones del mercado y la reglamentación de éste lo permite, una unidad de generación que no ha tenido participación importante en el despacho de energía por ser una unidad cara, puede mejorar su situación económica participando en el servicio de reserva.

Bajo la suposición de diferentes esquemas de compensación por el servicio de reserva se requiere el desarrollo de herramientas que le permitan a la GenCo realizar un análisis minucioso en la toma de decisiones a fin de evaluar que esquema y bajo qué condiciones maximiza sus ganancias. De los esquemas de pago para los servicios de energía y reserva presentados en este trabajo, el análisis realizado a través de la formulación Europea resulta más sencillo que el análisis realizado bajo la formulación Europeo-Americana.

De la formulación Europea se observa que el generador obtendrá mayores ganancias cuando el OS no le requiera la reserva, la opción no se ejerce. Lo anterior debido a que los costos de operación no incluyen la reserva. La evaluación de la opción Europea-Americana presenta un mayor grado de dificulta ya que requiere de la simulación de una contingencia a fin de representar el ejercicio de la opción Americana.

\section{AGRADECIMIENTOS}

Giselle Daniela Mendoza Mendoza, agradece al Consejo Nacional de Ciencia y Tecnología, CONACyT, por el apoyo económico recibido para la realización de estudios de maestría. 


\section{REFERENCIAS}

[1] Z. Xu, Z. Y. Dong and C. K. Pang. "Spinning Reserve Procurement in an Integrated Energy and Ancillary Service Market". The Australasian Universities Power Engineering Conference. Christchurch, New Zealand. 2003.

[2] P. Cramton, H. Chao and R. Wilson. "Review of the Proposed Reserve Markets in New England". University of Maryland, EPRI, and Stanford University. January, 2005.

[3] J.A. Quiñonez. "Aplicación de Modelos de despacho óptimo para la evaluación del servicio auxiliar de reserva de potencia activa". Tesis de Maestría en Ciencias. Instituto Tecnológico de Morelia. Morelia, México. 2006.

[4] J. Wang, X. Wang and Y. Wu. "Operating reserve model in the power market", IEEE Trans. On Power Systems, Vol. $20 \mathrm{~N}^{\mathrm{o}} 1$, pp. 223-229. February, 2005.

[5] S. Just and C. Weber. "Pricing of reserves: valuing system reserve capacity against spot prices in electricity markets", Energy Economics. Vol. $30 \mathrm{~N}^{\circ}$ 6, pp. 3198-3221. November, 2008.

[6] G.A. Bassotti, F. Olsina y F. Garcés. "Cooptimización de los mercados de energía y reserva considerando restricciones del sistema de transmisión”. Décimo Tercer Encuentro Regional Iberoamericano de CIGRÉ, Puerto Iguazú, Argentina. Mayo 2009.

[7] S. Myers. "Determinants of corporate borrowing". J. Fin. Econ. Vol. 5 Nº 2, pp. 147175. 1977.

[8] J. Pajares Gutiérrez, C. Hernández Iglesias y A. López Paredes. "Opciones reales en las decisiones de inversión”. II Conferencia de Ingeniería de Organización, Vigo. Septiembre, 2002.

[9] M. Paredes. "Entendiendo sobres opciones: financieras y reales". Instituto del Perú. Julio, 2009.

[10] C.E. Barría Quezada. "Inversiones bajo incertidumbre en generación eléctrica: aplicación de opciones reales y modelos de precios". Tesis de Magíster en Ciencias de la Ingeniería, Pontificia Universidad Católica de Chile. Santiago de Chile. Enero, 2008.

[11] R.M. Pringles, F.G. Olsina y F. Garcé. "Opciones reales en la evaluación de inversiones en mercados eléctricos competitivos". XII Encuentro Regional Ibero-americano de CIGRÉ Foz do IguazúPR, Brasil. Mayo 2007.

[12] S. Alonso Bonis, V. Azofra Palenzuela y G. de la Fuente Herrero. "Las opciones reales en el sector eléctrico. El caso de la expansión de Endesa en Latinoamérica". Universidad de Valladolid. Valladolid, España. Septiembre, 2008.

[13] J.L. Arriagada Carrazana. "Aplicación de Instrumentos Financieros en el Sector Eléctrico". Tesis para obtener el grado de Magister en Ciencias de la ingeniería. Pontifica Universidad Católica de Chile. Santiago, Chile. 2001.

[14] C. Gollier, D. Proultb, F. Thais and G. Walgenwitz. "Choice of nuclear power investments under price uncertainty: valuing modularity". Energy Economics, Vol. 27 No 4, pp. 667-685. July, 2005.

[15] R.M. Pringles, F.G. Olsina y F.F. Garcés. "Evaluación de inversiones en transmisión de energía eléctrica aplicando opciones reales". Décimo Tercer Encuentro Regional Iberoamericano de CIGRÉ, Comité de Estudio C1-Desarrollo de Sistemas y Economía. Mayo 2009.

[16] S.H. Martzoukos and W. Teplitz-Sembitzky. "Optimal timing of transmission line investments in the face of uncertain demand: an option valuation approach", Energy Economics, Vol. $14 \mathrm{~N}^{\mathrm{o}}$ 1, pp. 3-9. January, 1992.

[17] J.D. Saphores, E. Gravel and J.T. Bernard. "Regulation and investments under uncertainty: an application to power grid interconnection". Journal of Regulatory Economics. Vol. 25, pp. 169-186. 2002.

[18] K.W. Hedman, F. Gao and G.B. Sheble. "Overview of transmission expansion planning using real options analysis". Power Symposium, Proceedings of the $37^{\text {th }}$ Annual North American, pp. 497-502. 2005.

[19] D. Gardner and Y. Zhuang. "Valuation of Power Generation Assets: A Real Options Approach". ALGO Research Quarterly. Vol. $3 \mathrm{~N}^{\circ}$ 3, pp. 1-12. December, 2000.

[20] C. Tseng and G. Barz. "Short-term Generation Asset Valuation: A Real Options Approach". Operations Research, Vol. 50 N $^{\circ} 2$, pp. 297 310. March-April, 2002. 
[21] R.A. Jiménez Besoaín. "Metodología de Optimización Simultánea de Energía y Servicios Complementarios para el Despacho Económico". Tesis de Maestría en Ciencias, Pontificia Universidad Católica de Chile, Santiago de Chile. Diciembre, 2003.

[22] A. Pérez Villanueva. "Análisis y valoración de los mecanismos existentes para garantizar la seguridad del suministro Eléctrico. Adaptación al caso español”. Universidad Pontificia Comillas de Madrid. Junio 2006.

[23] E. Miguélez. "Gestión Centralizada de Restricciones Técnicas en Mercados de Energía Eléctrica. Aplicación al caso Español”. Tesis para la obtención del grado de Doctor. Universidad Pontificia Comillas de Madrid. Madrid, España. 2002.

[24] C. W. Richter and G.B. Sheble "A Profit-Based Unit Commitment GA for the Competitive Environment". IEEE Trans. On Power Systems. Vol. $15 \mathrm{~N}^{\mathrm{o}}$ 2, pp. 715-721. May, 2000.

[25] C. Weber. "Uncertainty in the electric power industry: methods and models for decision support". International Series in Operation Research and Management Science. 2004.

[26] J. Mun. "Real Options Analysis: Tools and Techniques for Valuating Strategic Investments and Decisions". John Wiley \& Sons. Inc. 2002. 